\title{
Dependence of Shock Mach Numbers upon the Shock-Vortex Interaction*
}

\author{
Fumio TAKAYAMA**
}

\begin{abstract}
A computer-based investigation has been conducted, regarding the interaction between a vortex ring, generated by starting a shock wave of Mach number 1.4, and plane shock waves of various shock Mach numbers. It is shown that a self intensification of shock waves takes place due to the mechanism of wave convergence by the interaction of shock wave and vortex. In order to evaluate the strength of intensification, the mean values of the maximum pressure and density for the time of interaction are calculated for the various shock Mach numbers. A remarkable feature observed during the computation is that the strength increases, then achieves a constant value, and finally decreases as the shock Mach number increases. These results show the typical features of interactions, such as the weak shock-strong vortex ring, a similar strength interaction of both shock and vortex, and a strong shock-weak vortex ring.
\end{abstract}

Key Words: Shock Wave, Vortex Ring, Shock-Vortex Interaction, Numerical Simulation, Shock Wave Convergence

\section{Introduction}

The interaction between shock waves and vortices is one of the fundamental issues of fluid dynamics which has been studied experimentally and theoretically over the past decade. Early studies focused primarily on the development of linear theories and their comparison with experimental results, and clarified an acoustic wave generated by a weak shock wave interacting with a columnar vortex ${ }^{(1)}$. In recent years, the studies have focused on the interaction between the strong shock and the vortex ${ }^{(2)}$ in relation to the noise generated by aircrafts. $\operatorname{Minota}^{(3)}$ has clarified experimentally, the deformation of shock waves in the case of an axisymmetric problem, and has stimulated considerable interest in the study of three-dimensional phenomena. The authors have numerically analyzed the deformation of shock waves observed in the experiment, and have observed a phenomenon of self-intensification by the convergence of shock waves due to the interaction ${ }^{(4)}$. We have also

* Received 2nd July, 2001. Japanese original: Trans. Jpn. Soc. Mec. Eng., (in Japanese), Vol. 66, No.648, B (2000), pp. 2028-2033 (Received 19th October, 2000)

** Department of Electronics and Computer Science, College of Science and Engineering, Iwaki Meisei University, Iwaki, Fukushima 970-8551, Japan. E mail : tkym@iwakimu.ac.jp shown the possibility of a self-ignition in reactive gases caused by this phenomenon ${ }^{(5)}$. More recently, a scattered wave generated by the interaction has been experimentally and numerically classified by the authors ${ }^{(6)}$. The shock-vortex interaction, which causes the shock wave convergence only by the fluid flow without the use of special devices like refractors, is an interesting phenomenon, but the relationship between the shock Mach numbers and the wave convergence is still not clear.

The objective of this study is to investigate the strength of self-intensification of various impinging shock waves interacting with a vortex ring, based on the previous study ${ }^{(4)}$. Governing equations based on the axisymmetric Navier-Stokes equations are computed by the Harten-Yee type TVD scheme ${ }^{(7)}$ using an adaptive multilevel grid refinement method ${ }^{(8)}$. The boundary conditions for the computation are set to be the same as those of the previous analysis.

\section{Governing Equations}

The governing equations are given by the following axisymmetric Navier-Stokes equations supplemented by the species conservation equation:

$$
\frac{\partial f}{\partial t}+\frac{\partial F}{\partial r}+\frac{\partial G}{\partial z}=\frac{\partial F_{v}}{\partial r}+\frac{\partial G_{v}}{\partial z}+\frac{H}{r} .
$$

Here, $f$ denotes $f=\left[\rho, \rho u, \rho v, \rho E, \rho_{i}\right]^{T} . F$ and $G$ are convection terms and $F_{v}, G_{v}$ and $H$ are transport 
terms. $E$ is the specific energy, $\rho$ is the density, $p$ is the pressure, $(u, v)$ are the axial and radial components of velocity and $\rho_{i}$ is' the density of species $i(i=$ 1 for oxygen, $i=2$ for nitrogen).

\section{Thermodynamic Properties and Transport Properties}

Since we use a computation code for the fluid flow fields including chemical reactions, the thermodynamic properties and the transport properties are denoted as follows. For the thermodynamic properties, the specific heat at constant pressure and the enthalpy of species $i$ are approximated by the polynomial form with the temperature $T$ as follows:

$$
\begin{aligned}
c_{p i} & =\frac{R^{0}}{M_{i}}\left(a_{1}+a_{2} T+a_{3} T^{2}+a_{4} T^{3}\right. \\
& \left.+a_{5} T^{4}+a_{6} T^{5}\right), \\
h_{i} & =\int c_{p i} d T=\frac{R^{0}}{M_{i}}\left(a_{7}+a_{1} T+\frac{a^{2}}{2} T^{2}\right. \\
& \left.+\frac{a_{3}}{3} T^{3}+\frac{a_{4}}{4} T^{4}+\frac{a_{5}}{5} T^{5}+\frac{a_{6}}{6} T^{6}\right) .
\end{aligned}
$$

where $M_{i}, R^{0}$ and $a_{l}$ are the molecular weight of species $i$, the universal gas constant and $l$ th polynomial coefficient ${ }^{(9)}$, respectively.

For the transport properties, the diffusion coefficient of each species is expressed by the mean binary diffusion coefficient between each species, considering the molecular transport of mass, energy and momentum. The transport coefficients, such as the mean binary coefficients between each species, viscosity coefficient and heat conductivity, are expressed as depending on the temperature ${ }^{(10)}$ using the ChapmanEnskog formula. The diffusion coefficient $D_{i}$ of species $i$ is given as follows:

$$
D_{i}=\frac{1-Y_{i}}{\sum_{j \neq i}^{2} \frac{X_{i}}{D_{i j}}},
$$

where the terms $D_{i j}, Y_{i}$ and $X_{i}$ represent the mean binary diffusion coefficient of species $i-j$, the mass fraction and mole fraction of species $i$, respectively.

The viscosity coefficient of the mixture is expressed as

$$
\mu=\sum_{i}^{2} X_{i} \mu_{i}
$$

Here $\mu_{i}$ is the molecular viscosity coefficient of species $i$. The thermal conductivity coefficient of the mixture is approximated as

$$
\varkappa=\left(\frac{c_{V}}{R}+\frac{9}{4}\right) \frac{R^{0}}{M} \mu,
$$

where $c_{v}, R$ and $M$ are the specific heat at constant volume, gas constant and molecular weight of the mixture, respectively.

\section{Numerical Method}

The governing equations are discretized using the
Harten-Yee type TVD scheme ${ }^{(7)}$ for the convection terms, and a central difference for the transport terms. For using the time splitting method the governing Eq. (1) is split into

$$
\begin{aligned}
& \frac{\partial f}{\partial t}+\frac{\partial F}{\partial r}=\frac{\partial F_{v}}{\partial r}+\frac{H}{r} \\
& \frac{\partial f}{\partial t}+\frac{\partial G}{\partial z}=\frac{\partial G_{u}}{\partial z} .
\end{aligned}
$$

The finite difference scheme of governing equations is given as follows:

$$
f^{n+2}=L_{z}(\Delta t) L_{r}(2 \Delta t) L_{z}(\Delta t) f^{n}
$$

where $L_{r}(\Delta t)$ and $L_{z}(\Delta t)$ are the TVD-central difference operators for Eqs. ( 7 ) and ( 8 ) for a time step $\Delta t$, respectively.

Computation grids for the numerical scheme are refined by the adaptive multilevel grid refinement method $^{(8)}$ because the field includes compact objects such as a shock wave, a vortex and a localized high intensity region. The grid refinement method permits the resetting of grid size from a first (rough $0.3 \mathrm{~mm}$ ) level to a third (fine $0.033 \mathrm{~mm}$ ) level and locally changing the grid level in response to the gradient of prefixed physical quantities. We also use the grid size of a fourth level $(0.011 \mathrm{~mm})$ to verify the numerical results.

\section{Boundary and Initial Conditions}

The upper half of a cylindrical tube is taken as the computing domain, shown in Fig. 1. The wall of the upper left side is set to a slip condition. The tube is filled with air composed of nitrogen and oxygen $\left(0.79 \mathrm{~N}_{2}+0.21 \mathrm{O}_{2}\right)$, initially at rest, the pressure $p_{0}=$ $101 \mathrm{kpa}$ and temperature $T_{0}=300 \mathrm{~K}$. A shock wave (shock wave $\mathrm{S}_{v}$ ) of nitrogen with Mach number $M_{V}$ is ejected through a hole on the left end wall of the cylindrical tube at $t=0$, which serves to generate a vortex ring. The right end of the tube is kept open, where a plane shock wave (shock wave $\mathrm{S}_{\mathrm{I}}$ ) of Mach number $M_{I}$ propagates to the left at $t=t_{l}$ and impinges on the vortex. In this study the Mach numbers of both shock waves are set to $M_{V}=1.4$ and $M_{I}=1.05 \sim 2.0$, with a step of 0.5 and also includes 1.225. The time $t=t_{I}$ is decided for the starting place

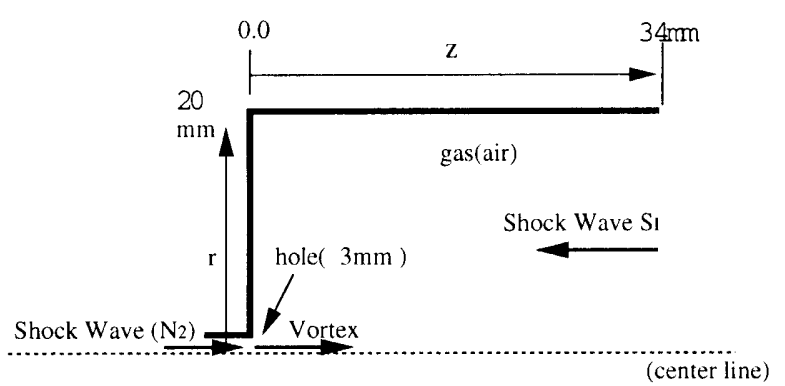

Fig. 1 Computational domain and boundary conditions 


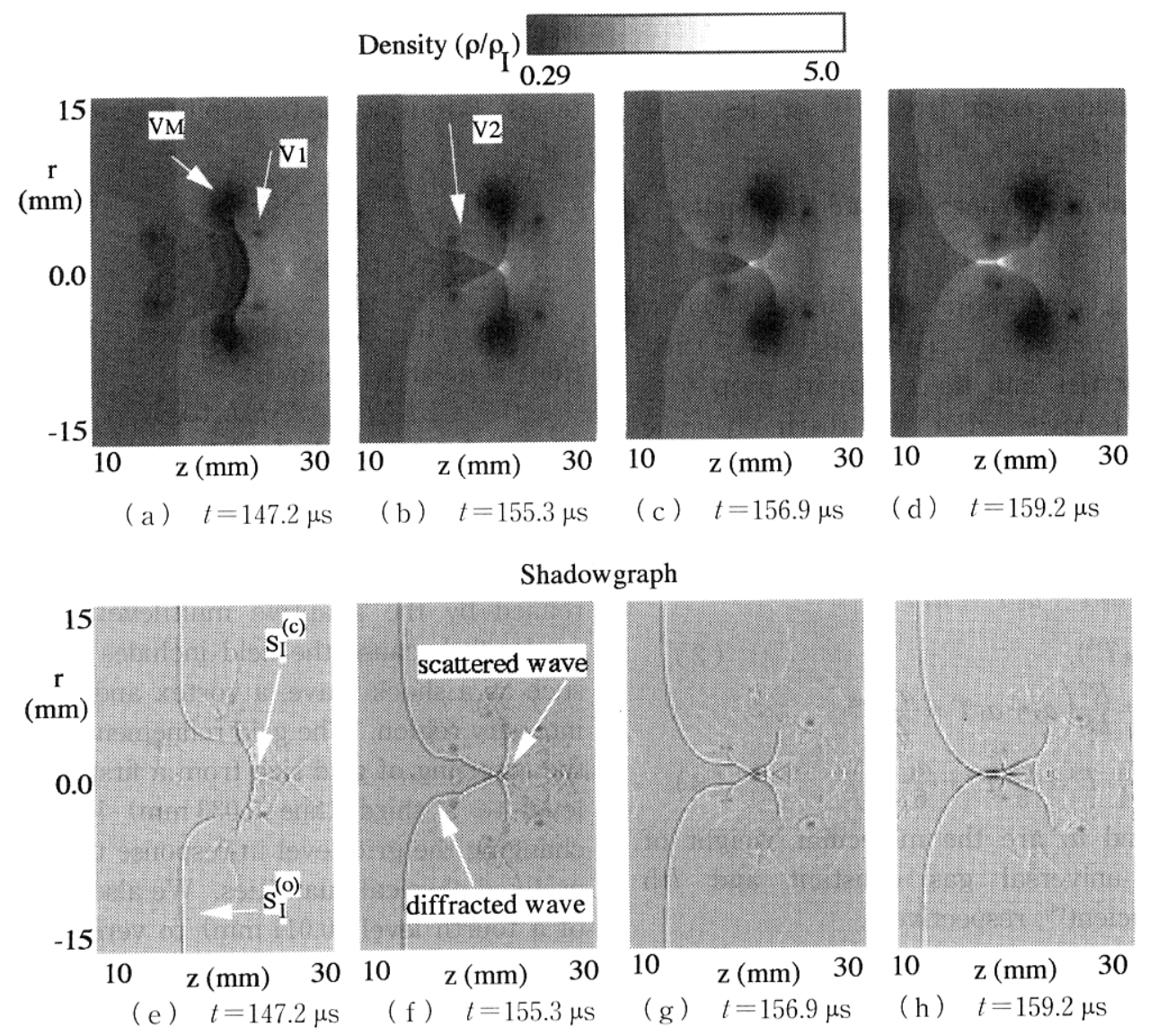

Fig. 2 Density distributions and computed shadowgraphs for shock Mach number $M_{l}=1.225$

of shock-vortex interaction to be equal with the various impinging shock waves.

\section{Results}

\section{1 Computer density diagrams and shadow- graphs}

Figure 2 shows computer density diagrams (Figs. 2( a $) \sim(\mathrm{d})$ ) and shadowgraphs (Figs. 2 ( e ) ( h ) ) for $M_{I}=1.125$ at four times during this computation. The lower half of this computer diagram is the mirror inversion of the upper half denoted by negative $r$-values. The density diagrams are black-white gray-scale plots with 256 levels normalized by the density $\rho_{I}$ of shock wave $\mathrm{S}_{I}$ as shown in the legend. The shadowgraphs are also gray scale plots of second derivatives of the density $\frac{\partial^{2} \rho}{\partial r^{2}}+\frac{\partial^{2} \rho}{\partial z^{2}}$ with 256 levels. In Fig. 2, we observe the following interesting features:

( i ) The main vortex (ring) $\mathrm{V}_{\mathrm{M}}$ generated by the shock wave $S_{v}$ and small vortices $V_{1}, V_{2}$ following it, progress to the right, while the impinging shock wave $\mathrm{S}_{\text {I }}$ propagates to the left and interacts with the vortex $\mathrm{V}_{\mathrm{M}}$; the wave $\mathrm{S}_{1}$ is deformed by the vortex flow, in such a way that the wave $S_{1}^{(c)}$ in the central part is retarded by the opposing flow of the vortex; a shock wave generated by the Labal nozzle mechanism of the vortex is observed in the central part of the main vortex ring; a triple point forms at the intersection of the diffracted wave and the retarded wave of the central part, and a Mach disk forms at the central part; the triple point also moves towards the $z$-axis (Figs. 2( a ) and (e )).

(ii) A scattered wave ${ }^{(6)}$, generated by the impinging shock wave interacting with the main vortex $V_{M}$, converges at the central part of the vortex and creates peak values of pressure and density; the wave $\mathrm{S}_{1}^{(0)}$ of the outer part is diffracted by the cooperating flow of the vortex and it propagates to the $z$-axis; a part of the diffracted wave is deformed by interacting with the second vortex $V_{2}$. The density at the convergent region of the scattered wave is about 3.5 times the density of shock wave $\mathrm{S}_{\mathrm{I}}$ (Figs. 2 (b) and ( f )).

(iii) The diffracted wave converges on the $z$-axis and creates the maximum peak value of density and attains a value about 5.0 times the density of shock wave $\mathrm{S}_{1}$ (Figs. $2(\mathrm{c})$ and $(\mathrm{g})$ ).

(iv) The diffracted wave deformed by the vortex $\mathrm{V}_{2}$ also converges on the $z$-axis and creates a peak value of density and attains a value about 4.6 times the density of shock wave $\mathrm{S}_{\mathbf{I}}$ (Figs. $2(\mathrm{~d})$ and $(\mathrm{h})$ ). 

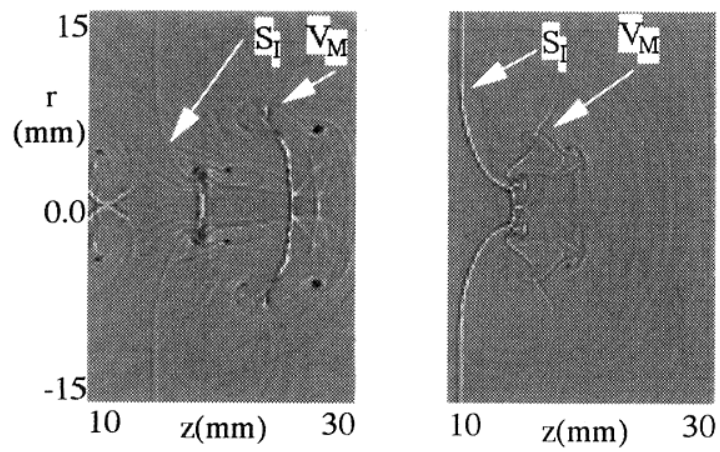

( a ) $M_{I}=1.05(t=162.95 \mu \mathrm{s})($ b $) \quad M_{I}=2.0(t=147.31 \mu \mathrm{s})$

Fig. 3 Computed shadowgraphs for shock Mach numbers $M_{I}=1.05$ and 2.0

The computed results have been compared with experimental shadowgraphs by Minota ${ }^{(3)}$ and the density contours by the authors ${ }^{(4)}$, and were found to be in good agreement. Furthermore, the process of deformation and the convergence of impinging shock wave due to interaction with the vortex ring are detailed clearly because of the high resolution of the computation grid. These results show that the generation of peak values of pressure and density in the local region is mainly due to two phenomena of the shock-vortex interaction, which are (i) the generation of a diffracted shock wave and its convergence; (ii) the generation of a scattered wave and its convergence.

The analysis of various Mach numbers $M_{I}=$ $1.05 \sim 2.0$ of the impinging shock wave has shown the generation of peak values of pressure and density by wave convergence for all Mach numbers except for Mach number $M_{I}=1.05$. Figure 3 shows computer shadowgraphs for the Mach numbers $M_{I}=1.05$ and 2.0. For $M_{I}=1.05$, the shock wave $\mathrm{S}_{1}$ cannot pass through the inner part of the vortex ring because of the strong opposing flow of vortex against the shock propagation, hence, the diffracted shock wave is not generated and the scattered wave also could not be detected clearly (Fig. 3( a )). While for $M_{I}=2.0$, the shock wave $\mathrm{S}_{1}$ can pass through the vortex ring, but the diffracted shock wave generated by the interaction does not converge on the $z$-axis because the deformation of the diffracted wave is very small in the case of the wave $S_{1}$ which has a high speed against the opposing flow in the vortex (Fig. 3(b )). However the generation of a scattered wave by the interaction and its convergence are observed, resulting in the small peak values of pressure and density.

\section{2 Time history of the maximum pressure and density}

Time histories of the maximum pressure $p_{M}$ and density $\rho_{M}$ normalized by pressure $p_{I}$ and density $\rho_{I}$ of the impinging shock wave for the Mach numbers $M_{I}=$
1.1, 1.225, 1.5 and 2.0 are shown in Fig. 4, where the horizontal axis shows a period of interaction. The period of interaction is defined as the time at which the maximum pressure $p_{M}$ satisfies $p_{M} / p_{I} \geq 3.0$, because $p_{M} / p_{I}$ in many cases shows gradual increases untill 3.0 and then boosts suddenly when shock-vortex interaction takes place. The interaction period shows a tendency to decrease as the shock Mach number $M_{I}$ increases and its average time is about $10 \mu \mathrm{s}$. Figure 4 (b) shows the time history of the maximum pressure and density corresponding to the density diagrams and shadowgraphs for the Mach number $M_{I}=1.225$ (Fig. 2). The diffracted shock wave converges at $t=$ $156.9 \mu \mathrm{s}$ (Figs. 2(c) and ( $\mathrm{g})$ ) and the normalized maximum pressure and density at that time attains peak values $p_{M} / p_{I}=10.0$ and $\rho_{M} / \rho_{I}=5.0$, respectively. Thus, the peak value for the time history of the maximum pressure and density corresponds to the convergence both of the shock wave and the scattering wave. Similarly, the time taken as a peak value of time history corresponds to the convergence of waves on the $z$-axis for the other Mach numbers $M_{I}=1.1,1.5$ and 2.0. The time histories of the maximum pressure and density for the Mach numbers $M_{I}=1.1 \sim 2.0$ can be classified into four phases depending on the impinging shock Mach number:

(i ) First phase: for Mach numbers such as $M_{I}=$ $1.1 \sim 1.15$, an average of the highest values for the history of the maximum pressure and density values reaches $\operatorname{Max}\left(p_{M} / p_{I}\right) \approx 7.0$ and $\operatorname{Max}\left(\rho_{M} / \rho_{I}\right) \approx 4.0$ respectively, although the history shows several isolated peak values, as shown in Fig. 4 ( a ).

(ii) Second phase: for Mach numbers such as $M_{I}=1.20 \sim 1.3$, an average of the highest values for the history of the maximum pressure and density values reaches $\operatorname{Max}\left(p_{M} / p_{I}\right) \approx 14.0$ and $\operatorname{Max}\left(\rho_{M} / \rho_{I}\right) \approx 5.5$ respectively, and the history shows a series of peak values by the connection of isolated peak values at the first phase, as shown in Fig. 4( b ).

(iii) Third phase: for Mach numbers such as $M_{l}=1.35 \sim 1.60$, an average of the highest values for the history of the maximum pressure and density values reaches $\operatorname{Max}\left(p_{M} / p_{I}\right) \approx 19.0$ and $\operatorname{Max}\left(\rho_{M} / \rho_{I}\right) \approx 7.0$ respectively, and the history shows a plot having one big peak value by the connection of several peaks at the second phase, as shown in Fig. 4( c).

(iv) Fourth phase: for Mach numbers such as $M_{I}=1.65 \sim 2.0$, an average of the highest values for the history of the maximum pressure and density values is $\operatorname{Max}\left(p_{M} / p_{I}\right) \approx 8.0, \operatorname{Max}\left(\rho_{M} / \rho_{I}\right) \approx 3.8$, respectively. The history shows a plot gradually changing from one big value at the third phase to two small isolated peak values, as shown in Fig. 4(d).

To summarize of these results, the maximum 


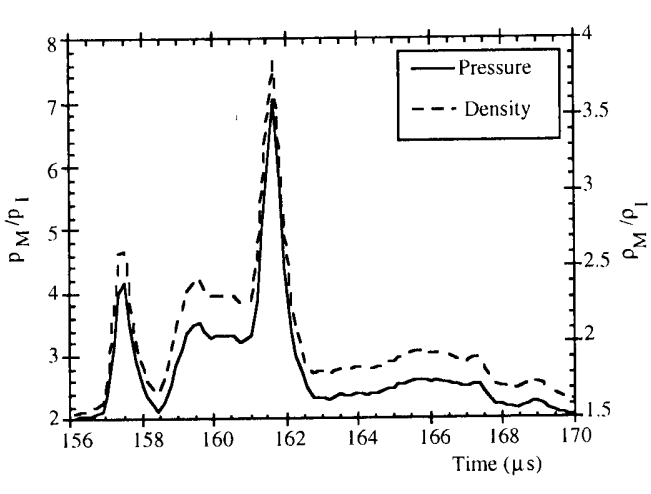

(a) $M_{I}=1.10$

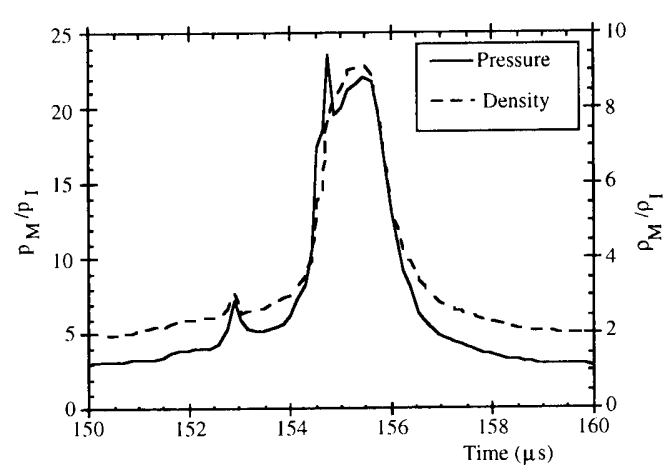

(c) $M_{l}=1.50$

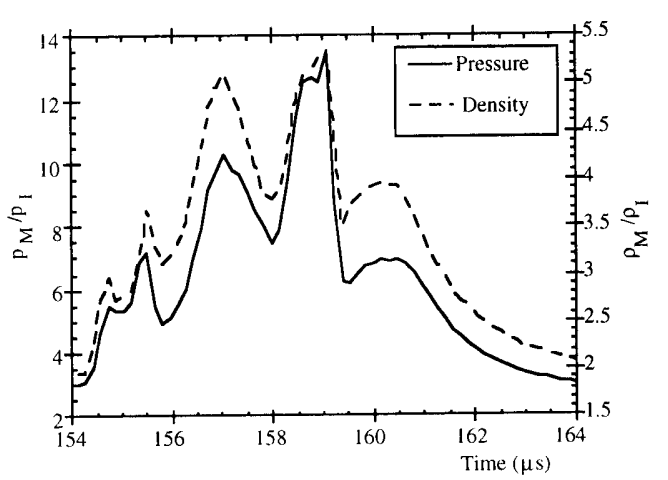

( b ) $\quad M_{I}=1.225$

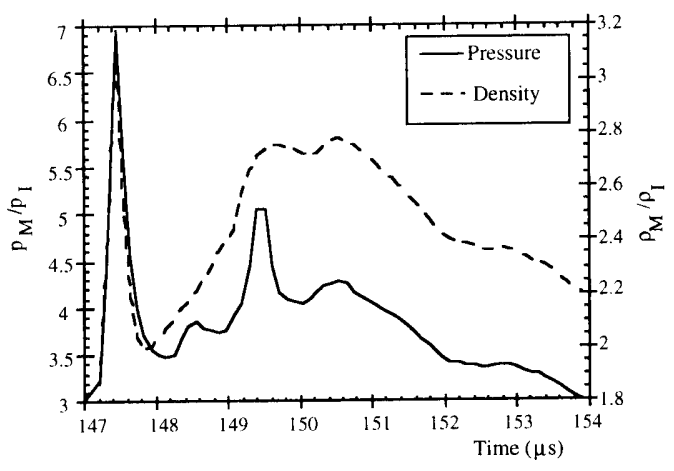

(b) $\quad M_{I}=2.00$

Fig. 4 Time history of maximum pressure and density in the interaction period normalized by the pressure and the density behind shock wave $\mathrm{S}_{1}$ for Mach numbers $M_{t}=1.1,1.225$, 1.5 and 2.0 , obtained using 3 -levels $(0.3,0.1,0.033 \mathrm{~mm})$ for the solution adaptive multilevel grid refinement method

value for the time history of the maximum pressure and density becomes higher as the shock Mach number increases and takes the highest value at the third phase, and then for larger Mach number $M_{I}$ the maximum value of time history decreases gradually. As shown by these phenomena and the results of the Mach numbers $M_{I}=1.05,1.225$ and 2.0 in section 6.1 (Figs. 2 and 3), the relative tendency of interaction as the shock Mach number increases, passes through a similar strength interaction of both shock and vortex from a weak shock-strong vortex interaction to a strong shock-weak vortex interaction. For the similar strength interaction the time histories of the maximum pressure and density attain the highest values for the ones with all interactions, and in this computation the Mach numbers $M_{I}=1.35 \sim 1.60$ (at the third phase) are regions of similar strength interaction. The reason why the shape of time history of the maximum pressure and density changes as the shock Mach number increases is that the propagating speed of the shock wave increases and the wave reaches the vortex earlier and begins to interact with it.

To investigate the validity of these numerical computations, the issues of shock-vortex interaction were computed using the solution adaptive multilevel grid refinement method using four levels, by further including the grid size of fourth level $0.011 \mathrm{~mm}$. As an example of the results, a time history of the maximum pressure and density for the impinging shock Mach number $M_{I}=1.225$ is shown in Fig. 5. By comparing this figure with Fig. 4 ( b), it is noted that both the shapes and peak values of the history are not in good agreement. Two reasons can be considered for the cause of this inconsistency. First, the grid size of computation could not be as small as the thickness of the shock wave. Second, the shock wave convergence by shock-vortex interaction has to take place ideally in order to use the axisymmetric model in the computation. If we compute the problem using a smaller grid size, it is considered that the shock wave convergence in the region of the grid size gives a higher value for the pressure and density due to the restriction of the axisymmetric model. This deduction is considered to be correct from comparison of the numerical results obtained by the three-level and four-level grid systems, where the maximum values of pressure and density are higher and the period of interaction 


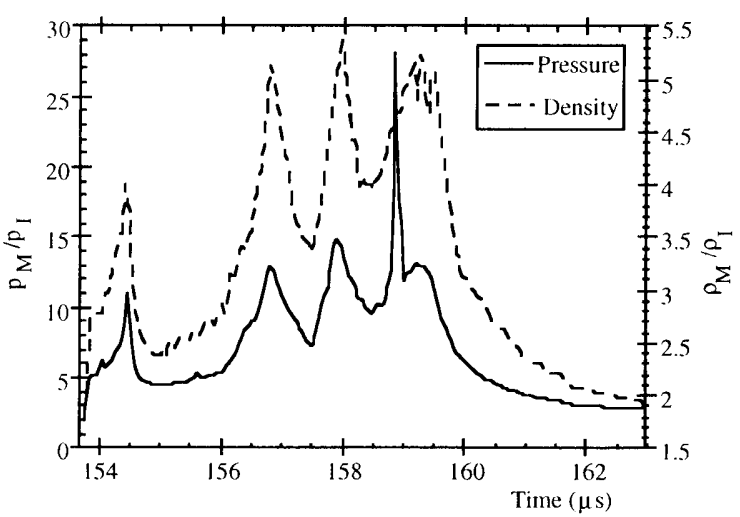

Fig. 5 Time history of maximum pressure and density in the interaction period normalized by pressure and density behind shock wave for shock Mach numbers $M_{I}=1.225$, obtained using 4 -levels $(0.3,0.1$, $0.033,0.011 \mathrm{~mm}$ ) for the grid refinement method

becomes shorter as the grid size decreases. Therefore, it is not possible to determine the peak value of shock wave convergence quantitatively using the axisymmetric model.

\subsection{Mean value of the maximum pressure and density}

Since our research is concerned with clarifying the relationship between the shock-vortex interaction for various shock Mach numbers and the intensification of shock wave convergence, we use mean values of $\tilde{p}_{M}$ and $\tilde{\rho}_{M}$ as the maximum pressure and density values between the period of interaction. The reason for using these mean values of maximum pressure and density is to utilize a numeric stable quantity to estimate the strength of convergence from the viewpoint of numerical results for the three-level and four level grid systems. The mean values of $\tilde{p}_{M}$ and $\tilde{\rho}_{M}$ are defined as

$$
\tilde{p}_{M}=\frac{1}{T_{21}} \int_{t 1}^{t 2} p_{M} d t, \quad \tilde{\rho}_{M}=\frac{1}{T_{21}} \int_{t 1}^{t 2} \rho_{M} d t,
$$

where $T_{21}$ is the period of interaction such as $T_{21}=$ $t_{2}-t_{1}, t_{1}$ and $t_{2}$ are the starting and terminating times of interaction, respectively.

Figure 6 shows the mean values of the maximum pressure and density normalized by pressure $p_{I}$ and density $\rho_{I}$, respectively, for $M_{I}=1.1 \sim 2.0$ on the threelevel (fine grid size $0.033 \mathrm{~mm}$ ) and four-level (fine grid size $0.011 \mathrm{~mm}$ ) computation grid. The pressure $\tilde{p}_{M}$ at the four-level grid is higher by about $10 \%$ in comparison with that at the three-level grid for each impinging Mach number $M_{I}$. The density $\widetilde{\rho}_{M}$ values at both grid levels almost correspond. Furthermore, the form of distribution of pressure and density almost corresponds for both grid levels also depending on the Mach number $M_{I}$. It is considered that the mean

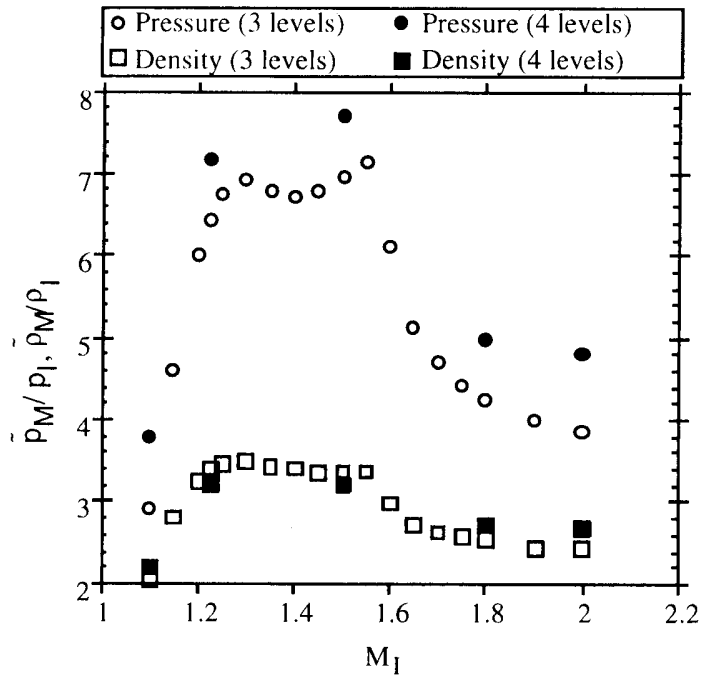

Fig. 6 Mean value of maximum pressure and density normalized by the pressure and the density behind shock wave $\mathrm{S}_{\mathrm{I}}$, obtained using both three-levels and four-levels for the grid refinement method

values of pressure and density are appropriate evaluation values for the intensification of convergence of shock waves due to the shock-vortex interaction in an analysis using the axisymmetric model.

The distribution form for the normalized mean values of the maximum pressure and density is divided into increasing, nearly constant and decreasing regions in proportion to the Mach number, which is considered to be a transition from weak shockstrong vortex interaction to strong shock-weak vortex interaction. The shock-vortex interaction for the Mach numbers $M_{I}=1.05 \sim 2.0$ is classified into five forms by considering the distribution in Fig. 6 and the analysis for the time history of the maximum pressure and density in the previous section, as follows:

(i) For Mach number $M_{I}=1.05$, the convergence does not take place; this means that the diffracted shock wave does not form because the impinging shock wave is unable to pass through the center of vortex ring due to the fast opposing flow of vortex ring; the form of interaction for the region of this Mach number is a very weak shock-strong vortex interaction.

(ii) For Mach numbers $M_{I}=1.1 \sim 1.15$, the mean value increases in proportion to the Mach number; the convergence of both the diffracted shock wave and the scattered wave is an isolated tendency because the impinging wave slowly passes through the center of vortex ring; the form of interaction for the region of these Mach numbers is a weak shock-strong vortex interaction.

(iii) For Mach numbers $M_{I}=1.20 \sim 1.3$, the time history of the maximum pressure and density takes a 
series of peak values by the effect of the interaction between the shock wave and both the main vortex and the vortices following it; this phenomenon causes a stronger shock wave convergence than that of the region (ii); the mean value of pressure is about 7 times and density about 3.5 times the values of the impinging wave, which are fairly constant, and the interaction period is about $10 \mu \mathrm{s}$; the form of interaction for this Mach number region is a low strength shock-strong vortex interaction.

(iv) For Mach numbers $M_{I}=1.35 \sim 1.6$, the interaction with the main vortex is dominant for the convergence rather than with the vortices following the main vortex and the time history of the maximum pressure and density takes one big peak value; the mean values for the pressure and density are fairly constant and almost the same as Mach number region (iii) ; the period of interaction is about $10 \mu \mathrm{s}$ and also similar to the previous region; the form of interaction in for this Mach number region is a strong shock-low strength vortex interaction.

(v) For Mach numbers $M_{I}=1.65 \sim 2.0$, the propagating speed of the impinging shock wave is fast because the shock wave is strong against the inner flow of vortex ring, hence, the convergence of triple point becomes slow and weak gradually in proportion to the shock Mach number $M_{I}$; therefore the mean values of pressure and density decrease depending on the increase of shock Mach number $M_{l}$; the form of interaction for this Mach number region is a strong shock-weak vortex interaction.

The results we have obtained are only for the case of the vortex generated by the shock wave with Mach number $M_{V}=1.4$, however it is considered that the tendency of shock-vortex interaction will not change because the impinging shock wave $S_{1}$ relatively exists as weak to strong waves against the shock wave $S_{v}$ even when the shock Mach number $M_{V}$ changes. Therefore the shock-vortex interaction is divided roughly into three tendencies through the similar strength interactions of both shock and vortex (including both the interactions of a low strength shock-strong vortex interaction and a strong shocklow strength vortex interaction) from the weak shock-strong vortex interaction (including the very weak shock-strong vortex interaction) to the strong shock-weak vortex interaction as the shock Mach number increases.

Subsequent interesting research could be conducted on the quantitative analysis of the dependency on the strength of the vortex for the shock-vortex interaction.

\section{Conclusions}

In this study, the strength of self-intensification by the convergence of shock waves due to the shockvortex interaction has been investigated for a vortex ring generated by a shock wave of Mach number $M_{V}=1.4$ and impinging shock waves of various Mach numbers $M_{l}=1.05 \sim 2.0$.

The shock-vortex interaction leads to the shock wave convergence, and by this effect high pressure and density are generated in the localized shock convergent region. The shock-vortex interaction mainly shows the interactions of three characteristic forms (such as a weak shock-strong shock interaction, a similar strength interaction of both shock and vortex and a strong shock-weak vortex interaction) as the impinging shock Mach number increases. The phenomena also include the effects of interaction not only with the vortex ring but also with the vortices follow. ing it.

\section{References}

(1) Weeks, T.M. and Dosanjh, D.S., Sound Generation by Shock-Vortex Interaction, AIAA J., Vol. 5 (1967), pp. 660-669.

(2) Ellzey, J.L., Picone, M. and Oran, E.S., The Interaction of Shock with a Compressible Vortex, NRL Mem. Rep. 6919, (1992).

(3) Minota, T., Interaction of Shock Wave with a Vortex Ring, Fluid Dyn. Res., Vol. 12 (1993), pp. 332-335.

(4) Takayama, F., Ishii, Y., Sakurai, A. and Kambe, T., Self Intensification in Shock Wave and Vortex Interaction, Fluid Dyn. Res., Vol. 12 (1993), pp. 343-345.

(5) Takayama, F., Kambe, T., Taki, S. and Sakurai, A., Spontaneous Ignition of a Reactive Gas Mixture by Shock-Vortex Interaction, Procs. of the 20th Int. Symp. on Shock Waves, (1995), pp. 10211026 .

(6) Tokugawa, N., Ishii, Y., Sugano, K., Takayama, F. and Kambe, T., Observation and Analysis of Scattering Interaction between a Shock Wave and a Vortex Ring, Fluid Dyn. Res., Vol. 21 (1997), pp. 185-199.

(7) Yee, H.C., Upwind and Symmetric Shock -Capturing Schemes, NASA T.M. 89464, (1987), NASA.

(8) Taki, S., Numerical Computation of Unsteady Flame Propagation, Procs. of 4th Symp. of Numerical Fluid Dynamics, (in Japanese), (1990), pp. 653-656.

(9) McBride, B.J. and Gordon, S., FORTRAN IV Program for Calculation of Thermodynamic Data, NASA TN D-4097, (1967), NASA.

(10) Strehlow, R.A., Fundamental of Combustion, (1968), p. 59, Int. Textbook Co. 